\title{
УДК 532.542:536.242 \\ ИССЛЕДОВАНИЕ СТРУКТУРЫ ТУРБУЛЕНТНОГО ПОТОКА ПРИРОДНОГО СЫРЬЯ В ТРУБОПРОВОДАХ С СЕКЦИЕЙ ПЕРЕМЕННОГО ПО ДЛИНЕ ПОПЕРЕЧНОГО СЕЧЕНИЯ КОНФУЗОРНО-ДИФФУЗОРНОГО ТИПА
}

\author{
Харламов Сергей Николаевич1, \\ kharsn@mail.ru \\ Фатьянов Дмитрий Сергеевич 1 , \\ dsf5@tpu.ru \\ 1 Национальный исследовательский Томский политехническийо университет, \\ Россия, 634050, г. Томск, пр. Ленина, 30.
}

\begin{abstract}
Актуальность работы вызвана необходимостью: понимания механизмов перестройки гидро-динамических, тепловых и диффузионных процессов, сопровождающих течение уәлеводородных вязких сред; уяснения характера их взаимодействия 8 ограниченных внутренних областях под действием комплексных эфффектов, сопровождающих течение в сложных трубопроводах; выработки эфффективных решений по оптимальному управлению транспорта природного сырья и повышения надежности функционирования энерго-напряженных узлов трубопроводов.
\end{abstract}

Объектом исследования являются пристеночные потоки капельных и газообразных углеводородных сред в конструктивных элементах внутренних систем, часто встречаемых в инженерных приложениях, в частности, нефттегазовой, химикотехнологической и тепло-энергетической отраслях промышленности. Такие элементы представляют собой участки коротких или протяженных трубопроводов с криволинейной поверхностью стенки в форме, подобной соединительным узлам или секциям переменного по длине поперечного сечения конфрузорно-дифффузорного типа. Причем течения в них осложнены эфрфектами влияния кривизны линий тока, пространственной деформацией, изменениями динамической структуры и давления.

Цель: уяснение неопределенностей, особенностей и закономерностей течений, а также возможности применения современных моделей турбулентности к прогнозу процессов в трубопроводах с секциями конфрузорно-дифффузорного типа; детальное исследование изменений «тонкой» структуры сложного сдвигового течения; выдача рекомендаций в практику расчета пристеночных потоков в указанных гидро-динамических конфигураций и интенсивных режимах работы оборудования.

Методы: теоретические и практические методы математического моделирования и численного исследования пространственных турбулентных течений, особенностей изменений их пульсационной структуры по схемам (моделям) с большим числом уравнений - RANS-модели турбулентности, включающие опорную динамическую двух-параметрическую базу. Выбор последней зависит от представлений: с какой степенью она способна воспроизвести статистические корреляции либо параметры сложного движения, локальные свойства (интегральный маситаб, кинетическую энергию, скорость ее диссипации и т. д.) вихря; учитывает ли неоднородный и анизотропный характер изменений структуры потока из-за деформационных эфффектов, возбуждаемых переменным давлением и градиентами рейнольдсовых напряжений.

Результаты. Проанализированы особенности турбулентных течений уәлеводородных вязких сред в трубопроводах, включающих соединительные элементы конфрузорно-дифрфузорного типа. Изучены возможности популярных в расчетах сложных сдвиговых течений вязких сред в трубопроводах с криволинейной границей стенки многопараметрические статистические модели турбулентности второго порядка, реализуемые в высоко- и низко-рейнольсовых версиях. Обнаружено, что лучшее описание интенсивности течений и размеров рециркуляционных зон в трубопроводах дают k-kiw/kL- u RSM-kL-модели, как более консервативные и устойчивые к возмущениям, идущим со стенок трубопровода. Поэтому включение их в опорную базу для RSM-модели вполне целесообразно. Расчетами выявлено существование второй зоны отрывного течения. Показано, что наибольшие изменения турбулентной кинетической энергии турбулентности, нормальных компонент тензора напряжений Рейнольдса локализованы в зоне взаимодействия сдвигового слоя с возвратным и прямоточным оторвавшимся течением, а генерация - вблизи точки отрыва. Соответствие расчетов структуры подобных течений данным других авторов позволяет утверждать, что понимание процессов, прогноз интенсивности динамических нагрузок в локальных зонах присоединения потока к стенкам по представленным моделям не приведет к аварийности фуннкионирования оборудования и обеспечит оптимальное управление транспортировкой сырья по трубопроводам.

\section{Ключевые слова:}

Математическое и численное моделирование, турбулентность, гидродинамика, пристеночное течение, трубопровод, конфузорно-дифффузорные соединения.

\section{Введение}

Повышение работоспособности трубопроводных систем связано с необходимостью детального мониторинга состояния оборудования, обеспечивающего транспорт продуктов на участках, осложненных влиянием климатических условий, рельефа местности, геометрией узлов и сочленений трубопроводов в форме участков с внезапным и/или последовательным расширением/сужением, поворотом, Т-образным со- единением, скачком площади поперечного сечения. При интенсивной прокачке природного сырья такое напорное оборудование испытывает большие динамические и тепловые нагрузки. Поэтому обеспечение безаварийного режима эксплуатации, долговечности и надежности трубопроводных систем требует постоянного мониторинга процессов, сопровождающих течение сред и устройств их управления. В указанных ситуациях экспериментальные исследования измене- 
ний структуры внутренних потоков крайне осложнены и затруднены отсутствием надежного оборудования, реагирующего на спектр возмущений в рабочей среде. Поэтому вопросы математического моделирования явлений и процессов переноса в сложных трубопроводах весьма актуальны с практической и фундаментальной точек зрения. Такие исследования позволяют проникнуть в суть механизмов и явлений, сопровождающих изменения «тонкой» структуры и интегральных параметров турбулентного потока сопротивления трения, интенсивность, длину и форму областей рециркуляционных движений. Поведение указанных переменных при сложном сдвиговом течении в трубопроводах с конфузорно-диффузорными участками, ответвлениями имеет важное техническое значение. Их корректный прогноз ценен для поддержания оптимального режима функционирования оборудования и в большой мере обусловлен выбором соответствующей модели турбулентности. Хорошо известно [1-10], что во внутренних турбулентных течениях в трубопроводах с соединениями и Тответвлениями, отличающимися переменной в местах соединения и по длине формой поперечного сечения, наряду с эффектами отрыва/присоединения возникают процессы, интенсифицирующие механизмы прямого и обратного перехода (реламинаризации). Они отличаются нетривиальным изменением компонент полного тензора напряжений Рейнольдса. Как сами по себе, так и в условиях отрыва такие устойчивые и переходные течения еще слабо изучены. Искривление поверхности трубопровода на диффузорноконфузорных участках, торможение и ускорение потока в его пристеночной области сопровождается возникновением вторичных течений, и это затрудняет их исследование в рамках простых схем. Кроме того, механизмы возникновения таких движений связаны с возбуждаемым давлением и сложным пространственным распределением компонент тензора напряжений Рейнольдса, в частности, его нормальных составляющих. Эти обстоятельства формируют ряд требований к выбору моделей турбулентности для расчета подобных течений. Опыт показывает [1-4, 11-13], что адаптация стандартных моделей к расчету рассматриваемой гидродинамической конфигурации требует кропотливой работы по выяснению их способности прогнозировать изменения в неоднородной анизотропной структуре турбулентности. В частности, исследования гидродинамики и теплообмена в трубопроводах с конфузорной областью при реламинаризации (в диапазоне изменений критерия ускорения Кэйса $-K_{c}=\frac{v}{\bar{U}^{2}} \frac{d \bar{U}}{d x}\left(K_{c}=(1 \ldots 20) \cdot 10^{-6}\right)$, детально исследованные в [1-4, 13], показали несовершенство отдельных моделей турбулентности, включающих понятие изотропной молярной вязкости, температуропроводности. Установлено, что более гибкими в анализе механизмов перестройки гидродинамических и тепловых процессов по длине трубопровода и изменения характера их взаимосвязи под действием ускорения/торможения, меняющихся по пространству градиента давления, компонент вектора осредненной скорости, кинетической энергии пульсационного потока, интегрального масштаба энергосодержащих вихрей и т. д., выступают двухпараметрические модели типа $(k L)$ - Г.С. Глушко [11], $(k \omega)$ - Ф. Ментера, Д. Уилкокса [12]. Это позволяет считать их надежной опорной базой для замыканий уравнений многопараметрических моделей турбулентности второго порядка с транспортными уравнениями для одноточечных корреляций пульсаций компонент вектора скорости полного тензора напряжений Рейнольдса (Reynolds Stresses Model (RSM)-модели). Однако, для подтверждения, что усовершенствованная версия RSMмодели с той или иной опорной базой будет корректна в численном моделировании рассматриваемых течений, необходимы надежные и детальные измерения распределений всего спектра параметров осредненного и пульсационного потока в области входа. Исследования сложных внутренних течений показывают $[1-4,13]$, что без этих данных на участках $x / D<5-8$ расчеты переоценивают пристеночный уровень пульсаций нормальных компонент тензора напряжений Рейнольдса на 10-15 \%, что приводит к завышенному уровню кинетической энергии турбулентности, а в неизотермических течениях - к завышенным значениям компонент удельного вектора турбулентного теплового потока. Такая ситуация с влиянием предыстории на структуру турбулентности значительно усложняет задачу расчета процессов в трубах с криволинейной границей и переходами вихревой природы по схемам с большим числом уравнений. Поэтому привлечение к анализу процессов сравнительно простых моделей будет способствовать решению проблем моделирования течений углеводородных капельных и газообразных химически однородных и неоднородных вязких гомогенных и гетерогенных сред в сложных трубопроводах.

Более того, при выборе модели турбулентности необходимо учитывать ее способность реагировать на мелкомасштабные процессы в узких пристеночных зонах. Такое внимание к вопросам эволюции микроструктуры турбулентности в сложных сдвиговых течениях диктуется контекстом проблем тепломассопереноса при течении углеводородных (УВ) смесей в трубопроводах. Представления о мелкомасштабной турбулентности целесообразно формировать по заключениям низкорейнольдсовых версий моделей, естественным образом учитывающих изменение локальных свойств вихря, в частности, интегральным масштабом энергосодержащих вихрей $(L)$, ответственных за обменные процессы [1]. В этом смысле обращение к схемам замыканий определяющих уравнений математической модели гидродинамики во внутренних системах (трубах, каналах) с транспортными уравнениями для кинетической энергии турбулентности $(k)$, интегрального масштаба $(L)$, предложенных российскими учеными Г.С. Глушко, О.Ф. Васильевым [11], и распространенных на широкий класс потоков, осложненных нестационарностью, неизотермичностью, действием массовых сил, химических реакций, фазовых переходов, а также их рас- 
четом вплоть до стенки, включая ламинарный подслой и буферную зону, представляется перспективHblм [1].

Другим важным с фундаментальной точки зрения вопросом анализа процессов переноса в мелкомасштабной структуре пристеночного течения в трубопроводах (ТП) и обращения к дифференциальному уравнению второго порядка для интегрального масштаба турбулентности, как двухточечной пространственно-временной корреляции между пульсациями компонент вектора скорости в двух разных точках пространства, являются проблемы выявления мелкомасштабных квазиупорядоченных структур на фоне стохастической составляющей турбулентности. Представление о турбулентности по поведению когерентных структур [14] как крупномасштабных упорядоченных образований и определение закономерностей в мелкомасштабных вихрях ставит вопрос о соотношении порядка и хаоса в сложном сдвиговом потоке [15-17].

Другими словами, использование $k L$-модели позволяет наряду с макроскопическим бесструктурным описанием привлекать идеи микроописания турбулентности как движения молей (вихрей), совершающих в потоке совокупные поступательновращательные движения. В такой трактовке турбулентные вихри предполагается анализировать как вращающиеся цилиндры, а изменения микроструктуры определять механизмами транспорта кинетической энергии турбулентности, в частности, ее диссипацией, которая распределена в потоке случайно, неоднородно и сосредоточена в зонах, примыкающих к минимальным размерам вихревого течения [1, 14-17] колмогоровским вихрям радиуса $\eta=\left(\frac{v^{3}}{\varepsilon}\right)^{0,25}$. Размер зоны диссипативных процессов можно определить по величине диссипативного микромасштаба турбулентности Тэйлора $(\lambda)$, порядок которой определен связью: $\eta<\lambda<L$, здесь $L-$ интегральный макроскопический масштаб турбулентности (размер системы, в которой происходит течение). Так как в макроскопическом подходе описания турбулентности диссипация энергии сосредоточена в основном в достаточно малых изолированных областях, то предполагается, что имеет место плотная упаковка диссипативных ячеек с проявлением порядка в микроструктуре.

При формулировке положений к моделированию процессов в сложном ТП отметим некоторые особенности течений с эффектами отрыва/присоединения потока к стенкам. Их расчет представляет серьезную проблему, поскольку вызывает значительные изменения интегральных параметров. Необходимы эффективные методы расчета, методики оценок динамических нагрузок, обусловленных отрывом/присоединением в случае сочленений конфузорно-диффузорного типа. Соответствующие экспериментальные исследования отмечают, что в диффузорной зоне поток с низкоскоростной стороны сдвигового слоя является сильно турбулентным. Непосредственно перед точкой присоединения сдвиговой слой резко искривляется, и часть потока уходит в рециркуляционную область под влиянием положительного градиента давления. Обнаружено, что в зоне присоединения проявляются стабилизирующие течение эффекты от: положительного градиента давления, кривизны линий тока и демпфирующего действия стенки, которые вызывают затухание нормального и касательного напряжений Рейнольдса в зоне присоединения. Поэтому течение в периферийной рециркуляционной зоне часто неустойчиво. Все это говорит о специфике течений в узлах расширения, а важнейшими параметрами процесса, формирующими длину присоединения, являются [18-22]: толщина отрывающегося пограничного слоя; турбулентность в ядре отрывающегося потока; градиент давления и относительная ширина канала. Поэтому анализ библиографических данных [23-25] приводит к отдельным замечаниям, характеризующим течения с отрыBом.

1. Все еще недостаточно материала для теста численных методов, особенностей процессов переноса из-за противоречивости положений и оценок результатов. Прогноз зон отрыва/присоединения требует дополнительного системного подхода и численных разработок.

2. Актуальны данные о природе крупномасштабных структур в зоне отрыва и присоединения.

3. В секциях расширения (например, течении за уступом) может отсутствовать традиционная область логарифмического закона стенки.

4. В течениях реализуется высоковязкой режим, в котором влияние турбулентных касательных напряжений мало по сравнению с влиянием молекулярных касательных напряжений, а пристеночные процессы сопровождаются анизотропными эффектами.

5. Турбулентность в пристеночной части не генерируется. Ее структура определяется характеристиками крупных вихрей, развивающихся над пристеночной областью и демпфирующим влиянием стенки. Течение в пристеночной области имеет ряд сходных с ламинарным течением черт, но не является ламинарным.

Таким образом, отмеченные особенности по детализации исследований структуры турбулентности, процессов и механизмов переноса в сложном сдвиговом потоке в трубопроводах с криволинейной границей поверхности стенки преследует ијель: 1) уяснить неопределенности, особенности, закономерности течений, а также возможности применения современных моделей турбулентности к прогнозу процессов в трубопроводах с секциями конфузорнодиффузорного типа; 2) детально исследовать изменения «тонкой» структуры сложного сдвигового течения; 3) дать разработчикам моделей обоснование к выбору направлений дальнейших исследований в отношении типа аппроксимаций, которые следует использовать для замыканий моделей и совершенствования имеющихся методик прогноза процессов в указанных гидродинамических конфигурациях и режимах работы оборудования. 


\section{Физико-математическая постановка задачи}

Предполагается, что трубопровод имеет сложную границу поверхности стенки, вследствие сопряжения предвключенной зоны $\left(X=x / h<L_{x}\right)$ постоянного поперечного сечения с соединительными переходными элементами размера $L_{x}$, геометрия которых напоминает узлы расширения/сужения конфузорнодиффузорного типа с тем отличием, что верхняя граница расчетной области (рис. 1) может быть как осевой линией (при анализе процессов в трубопроводах с плоской и цилиндрической симметрией), так и верхней стенкой. В области выхода $\left(X \geq L_{x}\right)$ ТП сохраняет форму своего поперечного сечения постоянной. Указанная особенность изменения образующей криволинейной стенки ТП связана с потребностью изучения закономерностей течений природного сырья в сложных ТП, а также накопления и уточнения теоретических сведений и экспериментальных данных о деталях гидродинамики и тепломассопереноса при течениях УВ вязких капельных и газообразных смесей во внутренних системах (в произвольных по форме поперечного сечения и режимам течений сред трубах, каналах, трубопроводах), которая создана сотрудниками Международной Научно-Образовательной Лаборатории (МНОЛ) «Нефтегазовая гидродинамика и тепломассообмен» при Инженерной школе Природных ресурсов НИ ТПУ. С учетом сказанного, принимается, что расчетная область ТП имеет форму, соответствующую заданию следующих параметров (рис. $1)$, где $h$ - высота уступа; $L_{x}, L_{y}$ - соответственно характерные продольный и поперечный размеры участка соединения. Движение углеводородных слабосжимаемых капельных и умеренно сжимаемых газообразных сред в трубопроводе считается установившимся, существенно дозвуковым (с числами Маха $\mathrm{M}<0,3)$, изотермическим, при отсутствии действия внешних сил и отвечает высоким значениям интенсивности турбулентности в области входа (степень турбулентности - Тu 1...10\%).

Инженерам-механикам хорошо известны проблемы получения экспериментальной информации об изменениях структуры течения УВ сред по ТП в режимах запуска/останова оборудования, сопровождающихся высокими нагрузками и нередко вызывающих аварийные ситуации. Управление и прогноз таких ситуаций требует, чтобы моделирование учитывало детали изменений спектра параметров, характеризующих турбулентность. Поскольку это невозможно для широких условий функционирования ТП, часто используется опосредованный подход, опирающийся на идеи подобия, аналогий процессов, сопровождающих сложное течение, учет их в исследовании конкретной тепло- и гидродинамической конфигурации. С целью обобщения/уточнения имеющихся данных о течении УВ сред в ТП с переходниками сложной формы $[1-4,13]$ в настоящей работе исследуются возможности отдельных моделей турбулентности и деталей расчета течений УВ сред в указанном ТП. При отсутствии экспериментальной информации полезными для валидации моделей и верификации ре- зультатов расчетов будут данные других исследований на аналогичной геометрии, например [24, 25], описывающих пристеночные процессы в криволинейных каналах по DNS/LES-подходам. Резюмируя сказанное выше, отметим, что моделирование течения осуществляется в условиях, характерных для транспорта природного сырья в ТП при выводе оборудования на оптимальную мощность в следующем диапазоне изменений чисел Рейнольдса $R e=(2,4 \ldots 15) 10^{3}$.

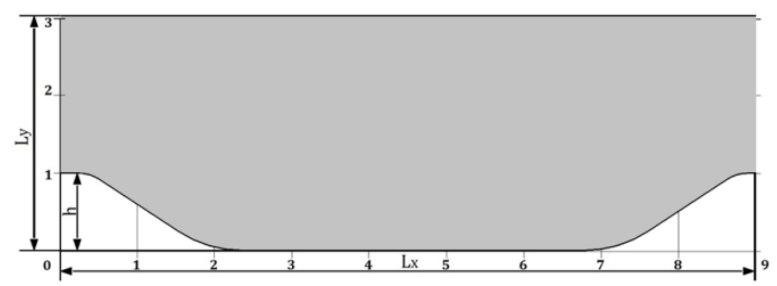

Рис. 1. Геометрия участка трубопровода с конфузорнодиффузорной зоной

Fig. 1. Geometry of pipeline section at confuser-diffuser zone

\section{Основные уравнения и соображения относительно модели турбулентности}

Система уравнений, определяющих течение однородных химически инертных вязких сред, включает уравнения законов сохранения: массы (неразрывности), импульса (полные уравнения Навье-Стокса, осредненные по Рейнольдсу), и имеет вид, который ниже для простоты записи выполнен в тензорной форме $[1,14,26]$ :

$$
\begin{gathered}
\frac{\partial \rho U_{j}}{\partial x_{j}}=0 \\
\frac{\partial}{\partial x_{j}}\left(\rho U_{i} U_{j}\right)=-\frac{\partial P}{\partial x_{i}}+\frac{\partial}{\partial x_{j}}\left(\mu \frac{\partial U_{i}}{\partial x_{j}}-\rho \overline{u_{i}^{\prime} u_{j}^{\prime}}\right)+V_{i} .
\end{gathered}
$$

В записи уравнений (1), (2) предполагается, что все величины осреднённые, черта над квадратичными членами пульсирующих величин означает осреднение по Рейнольдсу, $V_{i}=\frac{\partial}{\partial x_{j}}\left[\mu\left(\frac{\partial U_{j}}{\partial x_{i}}-\frac{2}{3} \delta_{i j} \frac{\partial U_{l}}{\partial x_{l}}\right)\right]-$ дополнительные к $\operatorname{div}(\mu \operatorname{grad} \vec{U})$ вязкие члены.

Как отмечалось выше, развитие течения в криволинейном канале в значительной степени зависит от природы и интенсивности взаимодействия пристеночных сдвиговых слоев. В таких потоках из-за присутствия областей отрыва/присоединения, рециркуляционных зон, обратных переходов следует ожидать нетривиальных эффектов в изменении локальных свойств турбулентных вихрей, сильной деформации «тонкой» структуры и роста турбулентной диффузии в напряжениях. Известно [18-23, 26], что в зоне присоединения диссипация кинетической энергии турбулентности уравновешивается не механизмами генерации, а за счет диффузии в непосредственной окрестности стенки в области взаимодействия сдвиговых слоев. Данные особенности механизмов переноса в 
сложном потоке диктуют требования к их моделированию на основе модели турбулентности и получению детальных количественных оценок характеристик осредненного течения и турбулентности в низкорейнольдсовой зоне узла соединения криволинейного канала.

С учетом сказанного, для замыкания осредненных по Рейнольдсу уравнений Навье-Стокса привлекались: однопараметрическая модель турбулентности П. Спаларта-С. Аллмареса (SA) [27], разработанная для применения в аэрокосмической отрасли и точного расчета характеристик течения вблизи стенки; двухпараметрическая $k \varepsilon$-модель турбулентности (Т. Ши, [28]) с пристеночной функцией Ф. Ментера [29], созданная для обхода отдельных недостатков $k \varepsilon$-модели и расширения границ ее применения на низкорейнольдсовые области; двухпараметрическая базовая $k \omega$-модель (Ф. Ментер, [30]); двухпараметрическая $k L$-модель (Г.С. Глушко, [11]), трехпараметрическая $k-k_{1} \omega$-модель (Д. Валтерс, [31]), учитывающая некоторые эффекты ламинарно-турбулентного перехода; четырехпараметрическая с эффектами перехода (SST) модель (Ф. Ментера, [32]), являющаяся одной из последних модификаций модели переноса сдвиговых напряжений (SST) и учитывающая явления, возникающие при ламинарно-турбулентном переходе; модель переноса компонент полного тензора Рейнольдсовых напряжений (RSM-kL) [1, 33]. В расчетах использовалась разностная сетка из четырехугольных элементов с количеством ячеек порядка 50000. Среднее значение параметра $\mathrm{y}^{+}$колеблется около 0,5 , максимальное его значение не превышает 1 , что вполне достаточно для описания пристеночных явлений в представленных режимах функционирования ТП.

Заметим, что большинство инженерных моделей RANS-метода, популярных в приложениях (например, двухпараметрические динамические $k L-, k \omega$-, $k \varepsilon$-, $k \tau$-модели), требуют согласования с данными экспериментальных исследований. В сложных течениях с неоднородной анизотропной структурой замыкания определяющих уравнений (1)-(3) целесообразно выполнять по RSM-моделям для компонент полного тензора напряжений Рейнольдса, которые незамкнуты и используют при формулировке своих неизвестных соотношения, включающие различные локальные параметры вихря. Такой путь моделирования имеет свои преимущества/недостатки (например, [1-4, 11-16]). В нашем случае для получения заключений о преимуществе того или иного подхода возможностей RANS-метода и его моделей, а также из-за отсутствия детальных экспериментов об изменении структуры потока полезно воспользоваться данными DNS-подхода (например, [25]), а для моделирования напряжений привести оригинальную и редко используемую в практике прикладных исследований низкорейнольдсовую версию $R S M-k L$ модели турбулентности. Детали ее формулировки подробно изложены, например, в [1]. И для простоты изложения ниже приведена ее тензорная форма $[1,14,26]$ :

$$
\begin{aligned}
& U_{k} \frac{\partial \overline{u_{i}^{\prime} u_{j}^{\prime}}}{\partial x_{k}}=\frac{\partial}{\partial x_{k}}\left[v \frac{\partial \overline{u_{i}^{\prime} u_{j}^{\prime}}}{\partial x_{k}}\right]+ \\
& +\frac{\partial}{\partial x_{k}}\left\{c_{s}^{\prime} f_{\mu} \frac{L}{\sqrt{k}}\left[\begin{array}{l}
\overline{u_{i}^{\prime} u_{l}^{\prime}} \frac{\partial \overline{u_{j}^{\prime} u_{k}^{\prime}}}{\partial x_{l}}+ \\
+\overline{u_{j}^{\prime} u_{l}^{\prime}} \frac{\partial \overline{u_{k}^{\prime} u_{i}^{\prime}}}{\partial x_{l}}+\overline{u_{k}^{\prime} u_{l}^{\prime}} \frac{\partial \overline{u_{i}^{\prime} u_{j}^{\prime}}}{\partial x_{l}}
\end{array}\right]\right\}+ \\
& +\left\{-\left[\overline{u_{i}^{\prime} u_{k}^{\prime}} \frac{\partial U_{j}}{\partial x_{k}}+\overline{u_{j}^{\prime} u_{k}^{\prime}} \frac{\partial U_{i}}{\partial x_{k}}\right]\right\}+R_{i j}-\varepsilon_{i j} \text {; } \\
& U_{k} \frac{\partial k}{\partial x_{k}}=\frac{\partial}{\partial x_{j}}\left(v \frac{\partial k}{\partial x_{j}}+c_{s k} f_{\mu} \overline{u_{i}^{\prime} u_{j}^{\prime}} \frac{L}{\sqrt{k}} \frac{\partial k}{\partial x_{i}}\right)+ \\
& +\left\{-\overline{u_{i}^{\prime} u_{k}^{\prime}} \frac{\partial U_{i}}{\partial x_{k}}\right\}-\tilde{c}_{D} \frac{k^{1.5}}{L} ; \\
& U_{k} \frac{\partial L}{\partial x_{k}}=\frac{\partial}{\partial x_{j}}\left(v \frac{\partial L}{\partial x_{j}}+c_{s L} f_{\mu} \overline{u_{i}^{\prime} u_{j}^{\prime}} \frac{L}{\sqrt{k}} \frac{\partial L}{\partial x_{i}}\right)+ \\
& +P_{L}+c_{L f} c_{2 L} k^{0.5}\left(1-\frac{L^{2}}{x_{n}^{2}}\right) \text {. }
\end{aligned}
$$

Система включает транспортные уравнения для компонент тензора напряжений Рейнольдса (3), опорную базу из уравнений для кинетической энергии турбулентности (4), интегрального масштаба энергосодержащих вихрей (5).

\section{Краевые условия и метод решения}

Выше отмечалось, что при расчетах развивающихся сложных турбулентных течений с выраженной пространственной деформацией и взаимодействия со стенкой ТП возникают серьезные проблемы, связанные с выбором начальных условий для «тонких» параметров во входном сечении. Особенно это касается задания согласованных распределений параметров: $k, L, \omega$, $\varepsilon, \tau, \overline{u_{i}^{\prime} u_{j}^{\prime}}, i, j=\overline{1,3}$. Попытки задавать этот спектр параметров по эксперименту проблематичны. Поэтому при исследовании практических случаев течений вязких сред приходится рассматривать более общую задачу - транспорт сырья с однородными распределениями, что способно вносить заметные погрешности в прогноз турбулентной структуры пристеночного течения, в частности, большого максимума кинетической энергии турбулентности, нормальных компонент тензора напряжений Рейнольдса на весьма протяженном продольном участке ТП [1]. При отсутствии необходимого экспериментального материала при построении численного решения задачи мы будем ориентироваться на указанную математическую технологию.

Для численного интегрирования эллиптических уравнений (2)-(5), соответствующих рассматриваемой конфигурации течения, требуется задать граничные условия на всех граничных поверхностях расчетной области. Принимается, что на границе вверх по потоку ( $X=0$ - входе) имеем равномерные профили всех зависимых осреднённых и пульсационных величин, в то же время на границе, расположенной вниз 
по потоку ( $X=L_{x}-$ выход), градиенты зависимых величин полагались равными нулю. Так как эти условия не могут в точности соответствовать рабочим условиям функционирования трубопровода при транспорте УВ среды конкретного состава, учитывается, что положение входа и выхода принимается таким, чтобы наложенные на параметры граничные условия оказывали по возможности малое влияние на течение в интересующей области посредством варьирования в расчетах значениями интенсивности и масштаба турбулентности. Для всех твердых границ криволинейной стенки ( $r=R-$ ТП с соединением) условия отсутствия движения для осредненных и пульсационных параметров: $U=\overline{u_{i}^{\prime} u_{j}^{\prime}}=0 ; k=L=w=\varepsilon=\tau=0$. На центральной линии: случай ТП с осевой симметрией $(r=0)$ - условия симметрии для всех величин, выражающиеся в равенстве нулю радиальных (поперечных) производных от искомых величин, кроме касательных компонент тензора напряжений Рейнольдса $\overline{u^{\prime} v^{\prime}}, \overline{v^{\prime} w^{\prime}}, \overline{u^{\prime} w^{\prime}}$, непосредственно обращающихся в нуль; случай ТП с твердой стенкой $-\left(Y=y / h=L_{y}\right)-$ условия прилипания потока (сквозной расчет от стенки до стенки). Последний вариант - более актуален, т. к. составляет данные обобщений имеющихся результатов исследований сложных внутренних течений в МНОЛ.

Численное решение транспортных уравнений (2)-(5) строится с использованием метода контрольного объема, базирующегося на известном полунеявном методе для связанных давлением уравнений (SIMPLE), предложенным C. Патанкаром [34]. Детали метода и алгоритм расчета подробно изложенные в [1], включают этапы, связанные с: построением сетки в расчетной области участка криволинейного канала; дискретизацией дифференциальных уравнений, сводящей их к своему конечно-разностному аналогу по схеме со степенным законом и противопотоковой разностной схеме - системе линейных алгебраических уравнений (СЛАУ) с искомыми величинами в узловых точках расчетной сеточной области, т. е. в центрах контрольного объема. Причем при получении разностных аналогов для осевой и радиальной компоненты вектора скорости используются смещенные сетки. Полученные дискретные аналоги рассчитываются методом переменных направлений, который использует на каждом своем осевом и радиальном шаге $(\Delta x, \Delta y)$ метод прогонки для решения СЛАУ с трехдиагональной матрицей коэффициентов. При итерационном процессе для ускорения или замедления изменений искомой величины привлекаются методы верхней или нижней релаксации, эффект от которых проявляется в усилении коэффициента при центральном расчетном узле. Опыт применения такой технологии показывает, что для ускорения сходимости расчета поля скорости осредненного движения коэффициент релаксации имеет значения порядка - 0,2...0,3, пульсационного (переменные модели турбулентности) - 0,6...0,8

\section{Результаты и их обсуждение}

Разработка расчетного метода для оценки динамических нагрузок в рассматриваемом узле соединения на интуитивном уровне их физического моделирования предполагает, что процессы, обусловленные отрывом/присоединением потока на диффузорном участке и ускорением при прохождении конфузорной секции, допускают удовлетворительное согласие с эффектами частных случаев течений в трубопроводах с коэффициентом поджатия/расширения и последующим развитием в зоне постоянного поперечного сечения. Подробный анализ результатов моделирования тепловой и динамической структуры, интегральных параметров гидродинамики и теплообмена, эффектов обратного перехода в указанных конифигурациях, ранее выполненный в $[1-4,13]$, позволяет утверждать, что при значениях критерия Кэйса $\mathrm{Kc}<\mathrm{Kc}_{\text {кр }}$ рассматриваемые в данной работе двухпараметрические $k L$-, $k \omega$-модели турбулентности вполне уместны, надежны и могут составлять опорные базы для RSM-моделей при прогнозе критических режимов анизотропных течений в ТП с реламинаризацией $\left(\mathrm{Kc}_{\mathrm{K}} \mathrm{Kc}_{\mathrm{\kappa р}}\right)$.

Более того, с целью обоснования надежности результатов моделирования течений в ТП с соединениями конфузорно-диффузорного типа в настоящей работе также выполнены количественные сравнения расчетов поля скорости потока и коэффициента трения на криволинейной стенке с результатами, полученными с использованием прямого численного моделирования (DNS) [25]. Заметим также, что канал с подобной геометрией, рассматривался в [24], где также отмечалось, что при использовании моделей турбулентности RANS-метода граница возвратного течения оказывалась значительно дальше, чем в эксперименте. Это демонстрирует недостатки полуэмпирического подхода, актуализирует исследования по предложению надежных схем замыкания определяющих уравнений и корректного прогноза эффектов в ТП с рециркуляционными потоками. Отдельные результаты, представленные на рис. 2-7, позволяют проникнуть в суть этих вопросов.

Картины изменений поля безразмерной осевой компоненты вектора скорости, нормированной на среднюю по поперечному сечению скорость во входной зоне ТП при турбулентном течении слабо сжимаемого газа в условиях численного эксперимента по DNS-методу, выполненному в [25], а также данных расчета настоящей работы с привлечением различных моделей RANS-метода, иллюстрирует рис. 2. На рисунке видны детали изменений структуры и точность ее прогноза моделями (серии картин Б-Ж) в сравнении с «эталонным» течением при $\mathrm{Re}=2800$ (рис. $2, \mathrm{~A}$ [25]). Для визуализации границ зоны формирования возвратного течения и локальной области точки присоединения потока $\left(X_{r}\right)$ по $[19,25]$ приведена общая линия $-X=X_{r}$.

Предварительная работа по адаптации моделей к расчетам сложных сдвиговых течений в ТП с конфузорной секцией показало, что результаты расчета динамической структуры весьма подобны для $k L$ - и $k$ $k_{l} \omega$-моделей, использующих схожий принцип моделирования многомасштабности вихрей переменными $L, k_{l} \omega$. Это позволяет надеяться, что их использование 
в качестве опорных баз в RSM-моделях при прогнозе сложных течений адекватно и удовлетворительно. Можно заметить, что модели SA [27], базовая $k \omega$ [30] и с эффектами перехода (transitional SST) [32] (рис. 2, Г-Е, соответственно), сдвигают точку присоединения течения намного дальше - до области начала сужения канала. Картина течения в отрывной зоне, рассчитанная $k \varepsilon$ [28], $k L / k-k_{1} \omega[1,11,31]$ и RSM- $k L-$ моделями [1] (рис. 2, Б, В и Ж, соответственно), оказывается близка к длине присоединения по DNS-методу.

Так, результаты расчетов по RSM-kL-модели (рис. 2, Ж) показывают, что в потоках с пространственной деформацией турбулентное поле в пристеночной зоне находится под влиянием взаимодействующих развивающихся сдвиговых слоев прямоточного и возвратного течений и сильно чувствительно к изменениям, вызываемым положительным градиентом давления в радиальном направлении от стенки. Особенности изменения поля скорости следует связывать с анизотропией вихревой вязкости, которая не является скалярной величиной (рис. 2, Б-Е), и ролью диффузионных процессов в переносе им- пульса. Также из структуры течения в конфузорной части соединения отчетливо видно, что ускорение потока приводит к уменьшению толщины вязкого подслоя и интенсификации перемешивания за счет механизмов турбулентной диффузии и перераспределения энергии из области ядра к стенке. Расчеты изменений интегрального масштаба турбулентности показывают, что в пристеночной конфузорной части он развивается интенсивнее и имеет значительно большие значения, чем в случае развивающего турбулентного пограничного слоя при обтекании плоской поверхности. Поэтому логарифмический закон распределения скорости, связанный с длиной пути смешения вблизи стенки, непригоден в форме, используемой при обтекании поверхности, и целесообразны исследования по модификации этого закона. Также предварительный анализ показывает, что и универсальная переменная закона стенки требует модификации на учет влияния эффекта кривизны подобно идеям [35], что может стать предметом будущих исследований эволюции «тонкой» структуры в сложном течении.
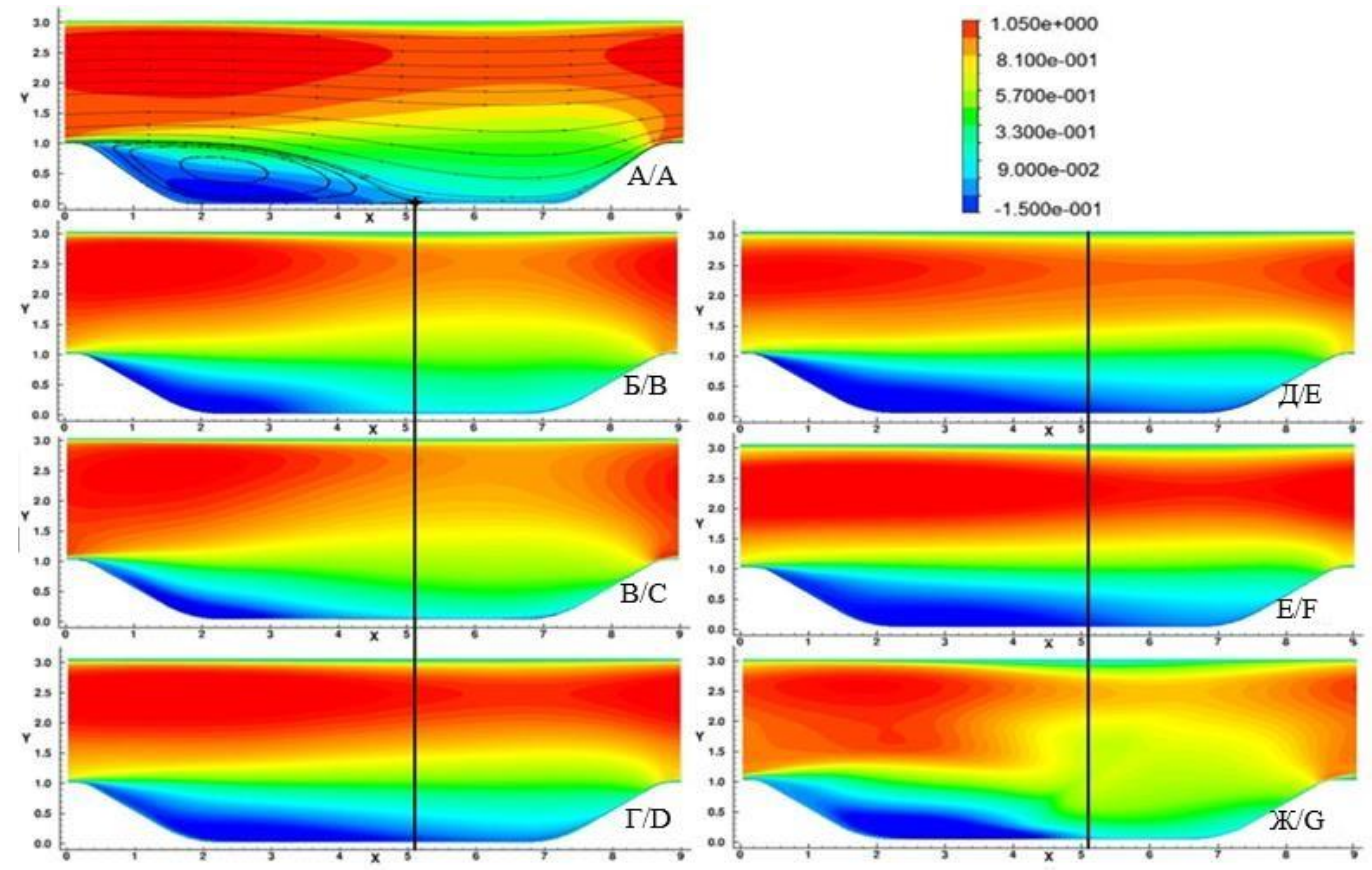

Pис. 2. Распределение поля безразмерной продольной компоненты вектора скорости, нормированной на среднюю скорость течения в сечении х=0. Данные расчета: A) DNS [25], Б) $k \varepsilon$ [28], B) $k L / k-k_{l} \omega[1,11,31]$, ) $S A$ [27], Д) базовая $k \omega[30]$, E) transitional SST [32], Ж) RSM-kL [1]

Fig. 2. Field distribution of the dimensionless longitudinal component of the velocity vector, normalized by the average flow velocity at section $x=0$. Calculation data: A) DNS [25], B) $\left.k \varepsilon[28], C) k L / k-k_{l} \omega[1,11,31], D\right) S A$ [27], E) based $k \omega$ [30], F) transitional SST [32], G) RSM-kL [1]

На рис. 3 представлены распределения коэффициента сопротивления трения $C_{f}$ по длине криволинейной стенки ТП. Выше отмечалось, что в классе двух параметрических отобранных моделей более успешна в сравнении с результатами DNS [25] группа $k L / k-k_{1} \omega$ моделей, т. к. с ее помощью точнее предсказано расположение границы зоны отрыва, а также наличие дополнительной отрывной области на участке $X \in[6,75,7,5]$. Поэтому и дальнейший анализ особенностей, закономерностей рассматриваемых течений связан с параметрами данных моделей как отдельно, так и в качестве опорной базы к RSMмодели тензора напряжений Рейнольдса. В частности, данные рис. 3 показывают, что за исключением $k \varepsilon$-модели прогноз интегральных параметров другими моделями неплохо согласуется с опытом. На этот эффект мы указывали, анализируя динамику пристеночных течений при небольших параметрах 
ускорения и загромождения потока. Также отмечали, что интенсификация изменений пульсационных параметров при увеличении кривизны поверхности стенки, режима течения, анизотропии турбулентности сопровождается потерей точности и проблематичностью их использования в расчетах реальных течений углеводородных сред с неизотермичностью, переменностью изменений теплофизических свойств, массовых сил, компонентного состава смеси и т. д. В указанных режимных параметрах более гибким инструментом выступает $R S M-k L$-модель напряжений. Отметим, что прогноз сопротивления в рециркуляционной области (рис. $3, X \in[2,5,4,5])$ говорит о более интенсивных возвратных процессах у стенки. Это подтверждает потребность коррекции низкорейнольдсовых формулировок ее членов, таких как корреляция пульсаций давления - скорость деформации, диссипации и турбулентной диффузии в течениях со слабой и умеренной анизотропией.

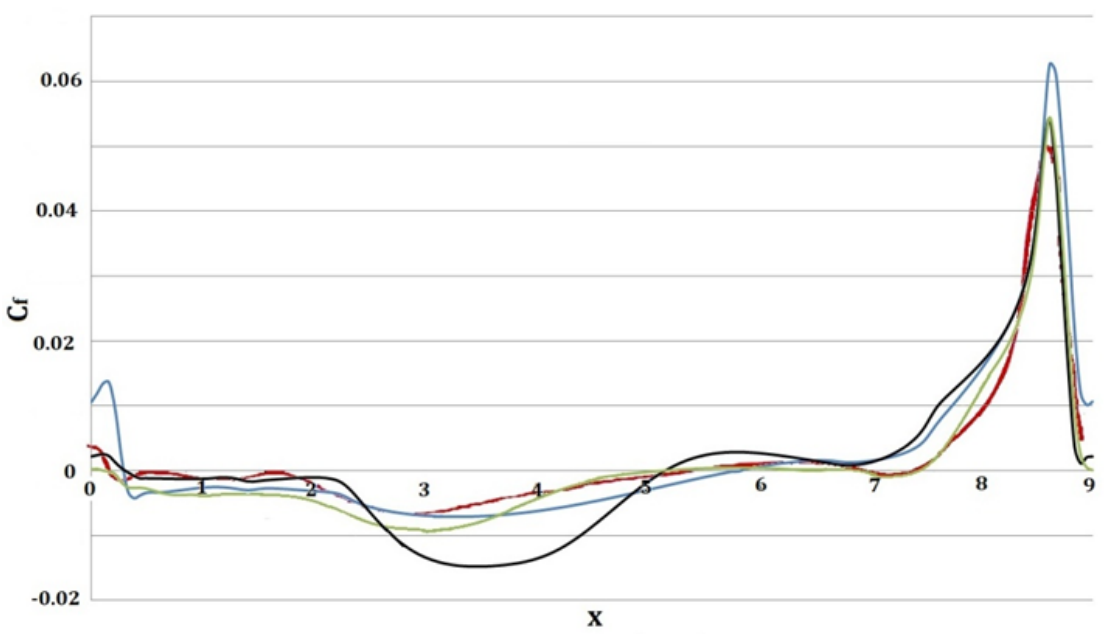

Рис. 3. Распределение коэффициента сопротивления трения $C_{f}$ по длине $(X=x / h)$ криволинейной стенки ТП. Расчеть отвечают течению в условиях численного эксперимента по DNS-методу при Re=2800 [25] с коэффициентом

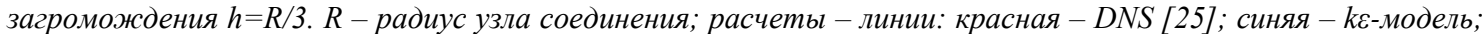
зеленая - $k L / k-k_{l} \omega$-модель; черная - RSM-kL-модель

Fig. 3. Distribution of friction resistance coefficient $C_{f}$ along the length $(X=x / h)$ of the curved wall of the PL. Calculations respond under conditions of numerical experiment on DNS-method at Re $=2800$ [25] with blocking up coefficient,

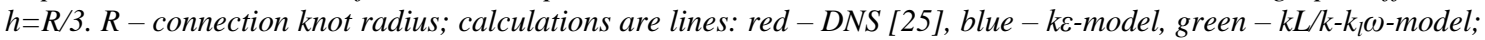
black-RSM-model

Рис. 4-7 иллюстрируют картины изменений локальных свойств турбулентного течения по $R S M-k L$ модели. В частности, на рис. 4 представлены распределения турбулентной кинетической энергии по каналу (A), ее механизмов (генерации (Б) - второй член в правой части (4) и диссипации (B) - третий член в правой части (4)). Стоит заметить, что данные механизмы наиболее важны в характеристике процессов локальной равновесности турбулентности в условиях развивающихся течений во внутренних системах постоянного поперечного сечения. Однако распределение производства, показанное на рис. 4, Б, подчеркивает нетривиальность механизмов изменения $k$ при течении в сложном трубопроводе. Из расчетов следует, что при отрывных процессах в пристеночной зоне диссипация (O(Dissipation) $10^{-4-5}$ ) уравновешивается диффузией, а не порождением $\left(O(\right.$ Generation $\left.) \sim 10^{0-1}\right)$.

Из расчетов рис. 4 видно, что максимальный уровень $k$ локализован в окрестности взаимодействия сдвиговых слоев оторвавшегося течения и возвратных рециркуляционных движений максимальной интенсивности. Взаимодействия повышенных значений поля напряжений Рейнольдса с градиентами осреденного потока в срывной зоне соединения ТП демонстрируют рост механизма порождения в общем энер- гетическом балансе изменений турбулентной кинетической энергии (рис. 4, Б). Особенности этого процесса сопровождает и подтверждает картина распределения диссипативного механизма изменения $k$ (рис. 4, В). Видно, что диссипация вихревого потока определенно организована. И это может служить полезной информацией при исследовании вопросов организации порядка в мелкомасштабной структуре турбулентности. Так, из рис. 4 , В, а также из данных распределений величины интегрального масштаба турбулентности $(L)$, его связи с диссипативным микромасштабом турбулентности Тэйлора $(\lambda)$ в этой области следует, что диссипируемая мощность на макроуровне равномерно и монотонно распределена по пространству. Можно видеть, что и на малых масштабах (в пределах масштаба $\lambda$, как характерной длины диссипации на микроуровне турбулентности) наблюдаются аналогичные процессы равномерного размазывания диссипируемой энергии турбулентности. Поэтому есть основания утверждать, что в хаотической случайной среде, каковой является турбулентное течение в ТП с криволинейной поверхностью, порядок проявляется в большом и малом. Порядок в большом проявляется в квазиупорядоченном расположении колмогоровских вихрей (структурность в 
случайной среде). Порядок в малом подразумевает упорядоченное течение в локальной окрестности колмогоровского вихря. Другими словами, вопрос о трактовке диссипативного микромасштаба диссипации Тэйлора $\lambda$ - это вопрос уровня описания структу- ры турбулизированной среды. Подразумевается [17], что на макроуровне $\lambda$ это эффективная длина диссипации турбулентной кинетической энергии, а на микроуровне - длина гидродинамического влияния процессов от колмогоровского вихря.
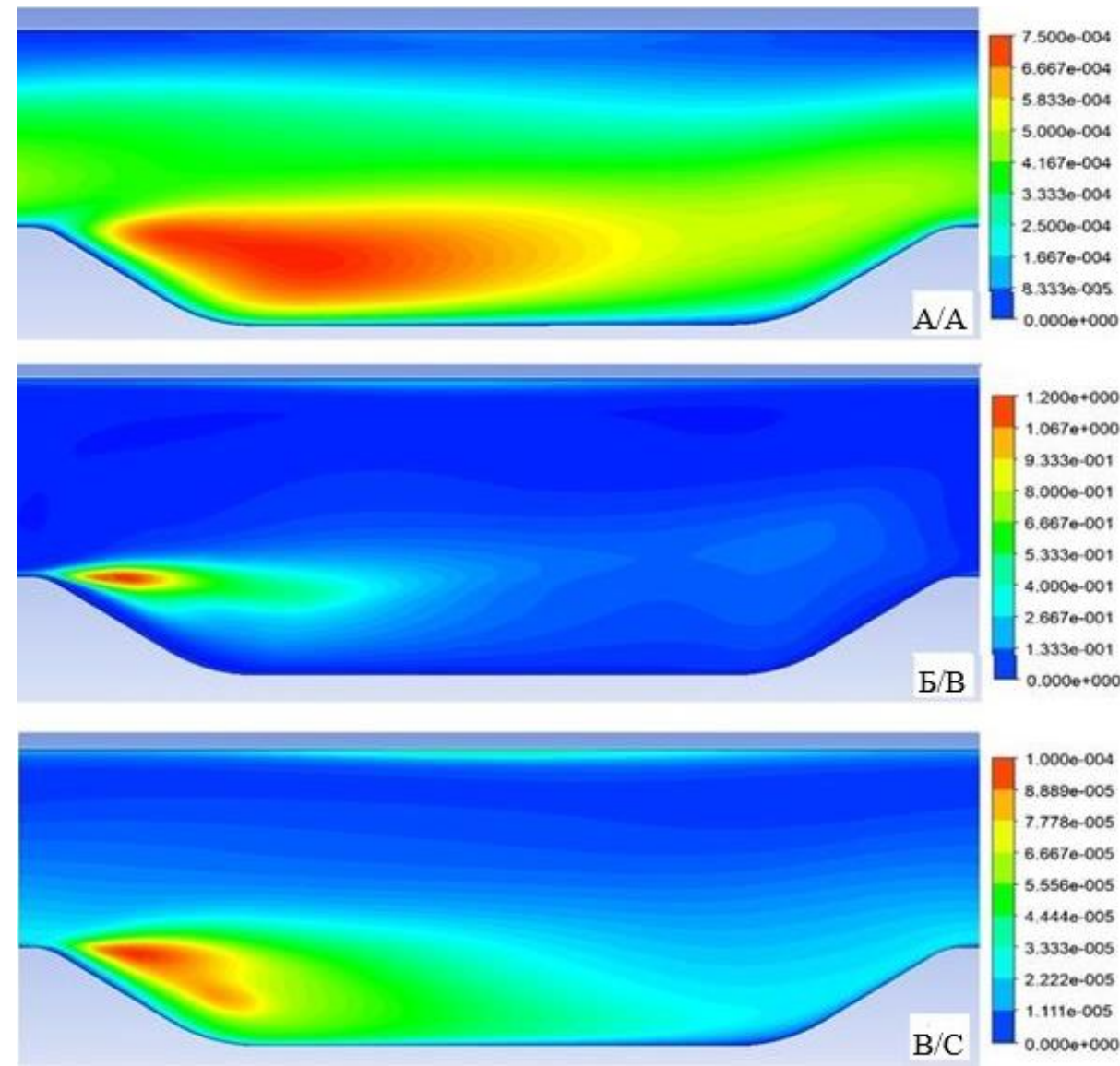

Pис. 4. Картины изменений абсолютных значений кинетической энергии пульсационного течения и ее отдельных механизмов переноса осредненным и пульсаиионным потоком в области участка соединения ТП: А) поле кинетической энергии турбулентности; Б) поле значений механизма ее генерации (второй член в (4)); B) поле диссипативных процессов кинетической энергии турбулентности (третий член в (4)). Расчеты выполнень при $R e=3600$ на форме ТП, представленной на рис. 2

Fig. 4. Patterns of changes of absolute values of kinetic energy of pulsating flow and its some transfer mechanisms by averaged and pulsating flow in the area of PL connected section: A) the field of turbulent kinetic energy; B) the field of values of its generation mechanism (second term, (4)); C) the field of dissipative processes of turbulent kinetic energy (third term, (4)). Calculations are performed at Re=3600 on the PL form shown in Fig. 2

На рис. 5 представлены картины особенностей, сопровождающих радиальные изменения абсолютных значений отдельных компонент тензора напряжений Рейнольдса (в частности, его нормальных компонент $-\overline{u^{\prime 2}}-A, Б, B$ - картины; $\overline{v^{\prime 2}}-\Gamma$, Д, $E$ - картины, доминирующих в отрывном потоке) в некоторых выделенных по пространству продольных сечениях $(X=0,1,2,4,8)$ указанной выше конфигурации ТП (рис. 2) при различных значениях $\operatorname{Re}(2800,5600,11200)$ при течении газообразного УВ вязкого изотермического потока.

По данным рис. 5 можно указать на некоторые особенности и закономерности турбулентности при обтекании стенок ТП. Так, при отрыве потока видно, что в слое смешения на границе взаимодействия с рециркуляционной областью наблюдается рост значений нормальных напряжений (сечения $X \in[0,2])$, а дальнейшее развитие потока приводит к обратному эффекту. Этот факт характерен и для течения в ТП с осевой симметрией. На верхней границе течение не имеет особенностей и демонстрирует наличие экстремума в буферной части пограничного слоя. Далее, по мере интенсификации течения (увеличения числа Рейнольдса), следует рост абсолютных значений рейнольдсовых напряжений, уменьшается толщина пристеночного слоя на верхней стенке, а также увеличивается относительный вклад как продольных $\left(\overline{u^{\prime 2}}\right)$, так и поперечных $\left(\overline{v^{\prime 2}}\right)$ напряжений Рейнольдса в области отрыва течения (сечения $X=0, X=1$ ) в общую картину их распределения по потоку. 

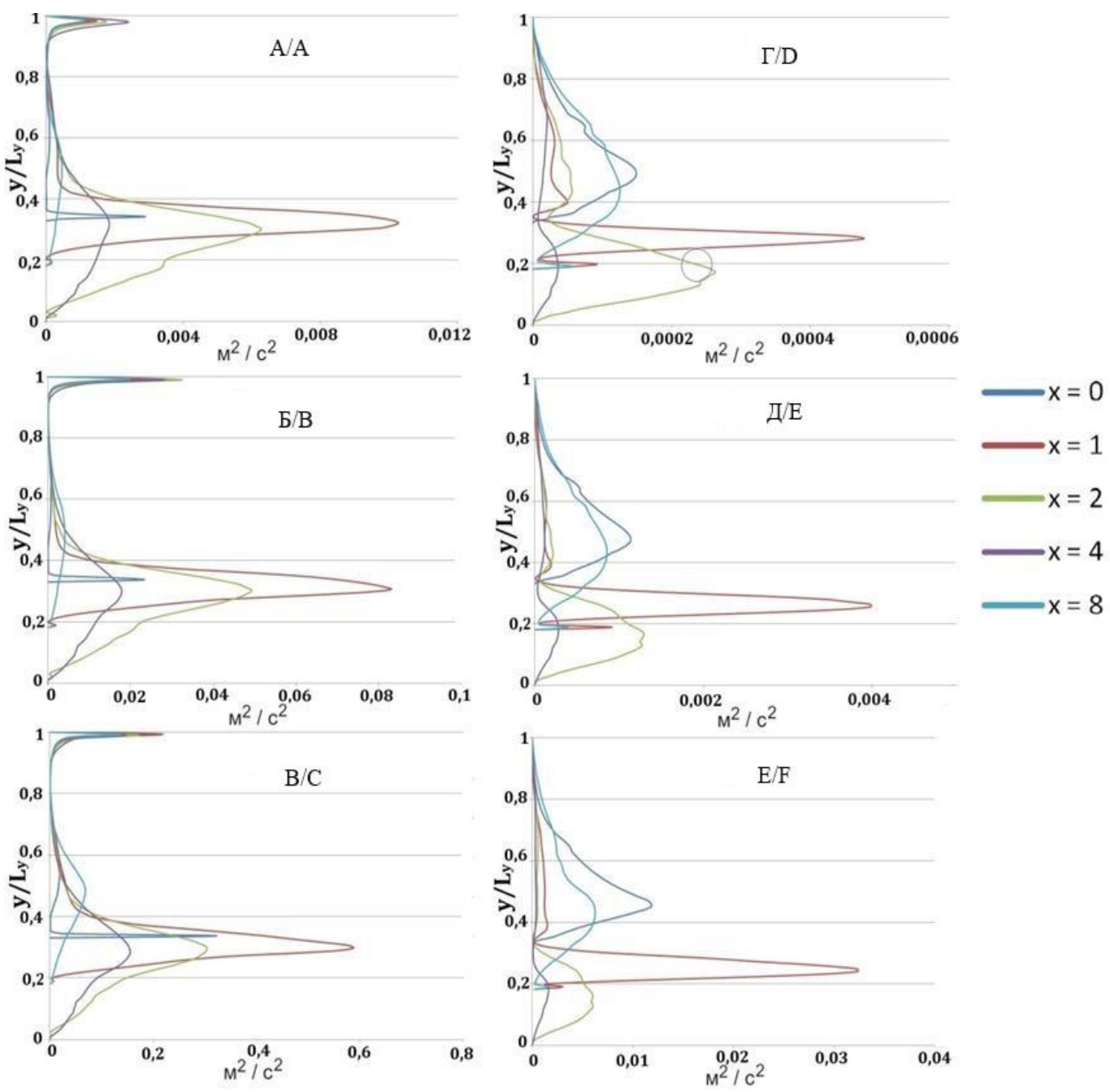

Рис. 5. Радиальные распределения нормальных компонент тензора напряжений Рейнольдса в некоторых выделенных по длине поперечных сечениях $(X=x / h)$ при различных значениях чисел $\left.\operatorname{Re} \overline{u^{\prime 2}}: A\right) \operatorname{Re}=2800 ;$;) $\left.\operatorname{Re}=5600 ; B\right)$ $R e=11200 ; \overline{v^{\prime 2}}:$ Г) $R e=2800$; Д) $R e=5600$; E) $R e=11200$

Fig. 5. Radial distributions of normal Reynolds stresses tensor components in some length-separated cross sections $(X=x / h)$ at different $R e . \overline{u^{2}}$ : A) $\left.R e=2800 ; B\right) \operatorname{Re}=5600$; C) $R e=11200 ; \overline{v^{\prime 2}}$ : D) $R e=2800$; E) $\left.R e=5600 ; F\right) \operatorname{Re}=11200$

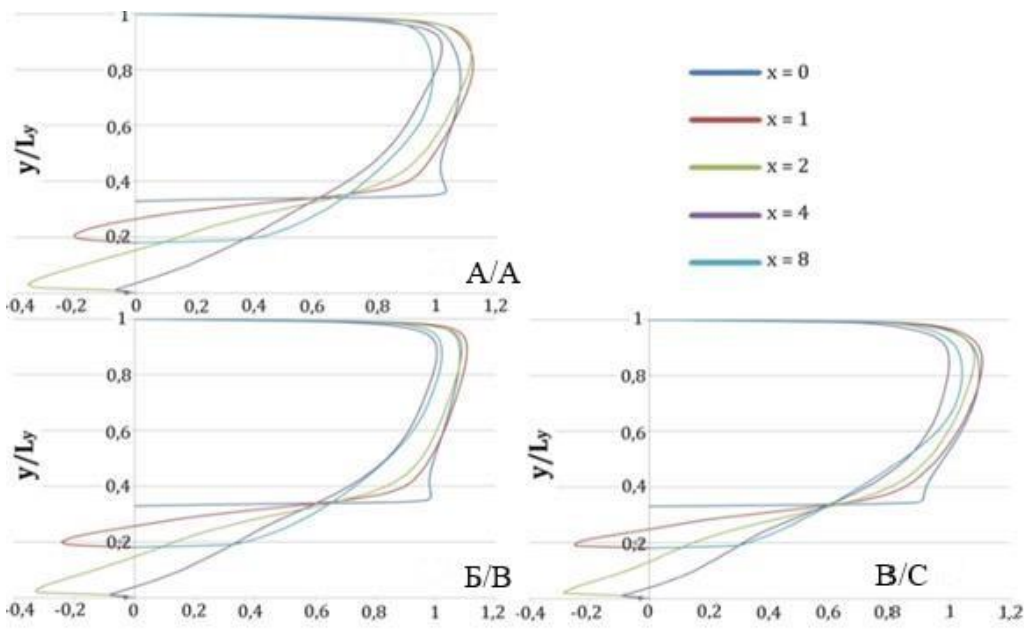

Рис. 6. Радиальные распределения безразмерной продольной компоненты вектора скорости, нормированной на среднюю скорость течения в сечении $X=0$, в различных сечениях по длине $(X=x / h)$. 3десь A) $R e=2800$; Б) $R e=5600$; B) $R e=11200$

Fig. 6. Radial distributions of the dimensionless longitudinal component of the velocity vector, normalized by the average flow velocity at section $X=0$ and at the different sections along the length $(X=x / h)$. Here: $A) \operatorname{Re}=2800 ; B) R e=5600$; C) $R e=11200$ 
Из рис. 6 следует, что с ростом числа Рейнольдса увеличивается абсолютная скорость возвратного течения. Видно, что скорость рециркуляционного течения относительно средней скорости потока интенсифицируется в сечении $X=1$, но уменьшается в сечении $X=2$. Отмеченные особенности поведения осредненного и пульсационного движения около криволинейной стенки ТП отражаются в изменении коэффициента сопротивления трения. Так, из рис. 7 следует, что максимальное значение коэффициента трения в сече- нии $X \approx 8,5$ снижается, исчезает вторая область возвратного течения. При входе потока в конфузорную зону рост значений сопротивления трения объясняется дополнительными эффектами ускорения течения в пристеночной части. Причем снижение $C_{f}$ в сечениях непостредственного выхода подтверждается изменениями структуры течения вследствие формирования области и условий для новых процессов деформации и отрыва вследствие ускорения. Такие режимы проанализированы в [1-4].

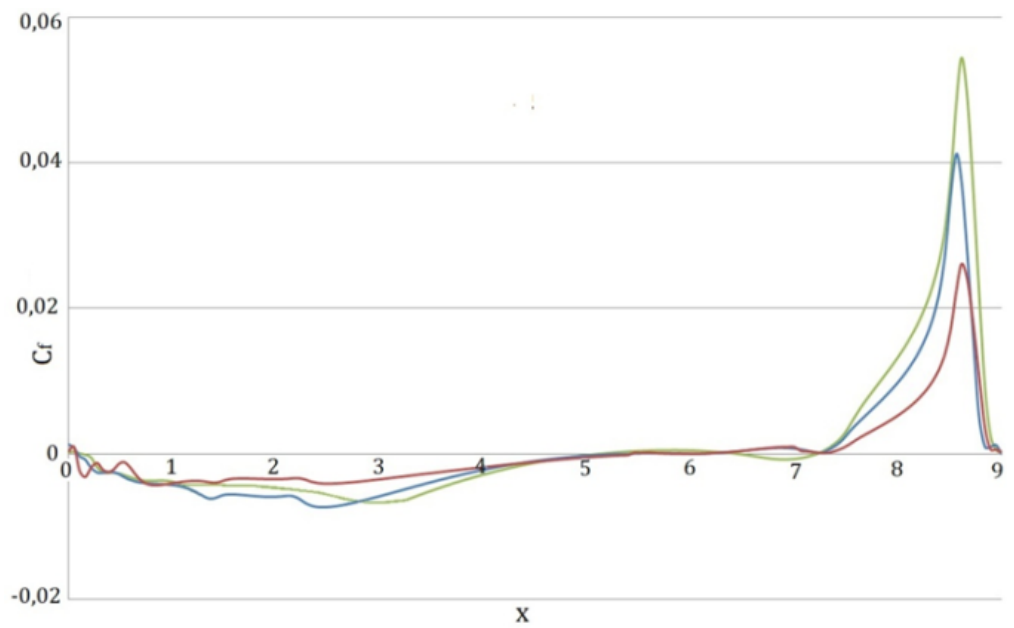

Pис. 7. Распределение коэффициента трения $C_{f}$ при различных Re по длине криволинейной стенки трубопровода. Расчеты -линии: зеленая $-R e=2800$, синяя $-R e=5600$, красная $-R e=11200, X=x / h$

Fig. 7. Distribution of the coefficient friction $C f$ at different Re along the length of the curved pipeline wall. Calculations are lines: green $-R e=2800$, blue $-R e=5600$, red $-R e=11200, X=x / h$

\section{Заключение}

Проведена серия расчетов, позволяющих проанализировать турбулентные течения УВ вязких сред в трубопроводах, включающих соединительные элементы конфузорго-диффузорного типа, сопряженные с участками постоянного поперечного сечения в его проксимальной и дистальной частях, верхняя стенка которого представляет собой либо линию осевой симметрии, либо прямолинейный участок поверхности. В последней гидродинамической конфигурации исследуются особенности, закономерности течений при различных значениях интенсивности режимов транспорта УВ по ТП $\left(\mathrm{Re}=(2,4 \ldots 15) 10^{3}\right)$ с целью уяснения механизмов, сопровождающих течение, а также неопределенностей, вносимых различными моделями турбулентности RANS-метода в прогноз изменений интегральных параметров (сопротивления трения), локальной структуры осредненного и пульсационного движения $\left(U_{i}, k, L, \omega, \varepsilon, \tau, \overline{u_{i}^{\prime} u_{j}^{\prime}}, i, j=\overline{1,3}\right)$ в пристеночной области соединения с криволинейной поверхностью.

Из данных численного моделирования можно сделать несколько выводов о возможностях отобранных моделей турбулентности RANS-метода, популярных в приложениях, в частности, $k \varepsilon$ [28], базовой $k-\omega$ [30], SA [27], с эффектами перехода (transition SST) [32],

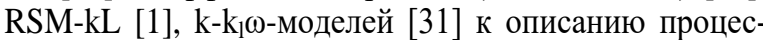
сов в ТП.
1. При сравнении картин изменений поля продольной компоненты вектора скорости, коэффициента сопротивления трения с результатами прямого численного моделирования обнаружено, что $k L$-, $k-k_{l} \omega$-модели наиболее точны в определении границ отрывного течения и могут составить перспективную опорную базу для многопараметрических моделей второго порядка для полного тензора напряжений Рейнольдса $(R S M-k L)$. Кроме того, указанные модели показали способность учета процессов в локальной зоне течения со вторым отрывом $(X \in[6.75,7.5])$. Опыт расчета показывает, что эти выделенные модели востребованы при прогнозе потоков с неоднородной анизотропной турбулентностью слабосжимаемых УВ сред в сложных ТП.

2. Сравнения с экспериментальным материалом реальных течений УВ сред в сложных ТП затруднены следующими недостатками. Первый связан с необходимостью задания согласованных начальных данных по всему спектру осредненных и пульсационных параметров течения во входном сечении $\left(U_{i}, k, L, \omega, \varepsilon, \tau, \overline{u_{i}^{\prime} u_{j}^{\prime}}, i, j=\overline{1,3}\right)$. Поля турбулентности, особенно напряжения Рейнольдса, не задаются должным образом и вносят погрешности в сравнении реальным процессом, превышающим уровень пульсационного течения (порядка 15 \%) на длинах (5-10)d. 
3. Особенности распределений кинетической энергии турбулентности $(k)$, скорости ее диссипации $(\varepsilon)$, величины интегрального масштаба энергонесущих вихрей $(L)$, изменений напряжений Рейнольдса $\left(\overline{u_{i}^{\prime} u_{j}^{\prime}}, i, j=\overline{1,3}\right)$, поля продольной компоненты вектора скорости $\left(U_{x}\right)$, коэффициента сопротивления трения $\left(c_{f}\right)$ на криволинейной стенке и оценки влияния на них фактора интенсификации режима течения из-за увеличения числа Рейнольдса $(\mathrm{Re})$ показывают, что важнейшими моментами, определяющими поведение течения, являются особенности во взаимодействии сдвиговых слоев прямоточного и возвратного потоков в условиях отрыва/присоединения потока к стенке в диффузорной области соединения и ускорения течения (увеличения критерия Кэйса, Kc) в конфузорной части.

4. Турбулентность определяется сложным балансом следующих механизмов: порождением энергии осредненным и пульсационным течением; диффузией турбулентности в радиальном направлении; переносом энергии между компонентами поля скорости, диссипацией. Следует отметить, что преимущественные изменения кинетической энергии турбулентности, ее отдельных механизмов (порождение, диссипация) локализованы в сдвиговом слое отрывной зоны и рециркуляционной области. В пристеночной части криволиней-

\section{СПИСОК ЛИТЕРАТУРЫ}

1. Бубенчиков А.М., Харламов С.Н. Математические модели неоднородной анизотропной турбулентности во внутренних течениях. - Томск: Томский государственный университет, 2001. $-448 \mathrm{c}$.

2. Kharlamov S.N., Alginov R.A. Specific aspects of turbulent flow separation in nods and junctions of pipelines // IOP Conference Series: Earth and Environmental Science. - 2014. - V. 21. - P. 1-5.

3. Харламов С.Н., Альгинов Р.А. Валидация статистических моделей второго порядка и методов численного расчета динамической структуры закрученных турбулентных течений в трубопроводах // Известия вузов. Физика. - 2010. - Т. 53. № 12/2. - C. 267-275.

4. Фатьянов Д.С. Термодинамические характеристики течения газа в трубопроводе с конфузорной секцией // Проблемы геологии и освоения недр: Труды XXI Международного научного симпозиума студентов и молодых ученых им. академика М.А. Усова. - Томск: Томский политехнический университет, 2017. - T. 2. - C. 236-239.

5. Теплообмен в дозвуковых отрывных потоках / В.И. Терехов, Т.В. Богатко, А.Ю. Дьяченко, Я.И. Смульский, Н.И. Ярыгина. - Новосибирск: Изд-во НГТУ, 2018. -247 с.

6. Howard R.J.A., Serre E. Large eddy simulation in Code_Saturne of thermal mixing in a $\mathrm{T}$ junction with brass walls // International Journal of Heat and Fluid Flow. - 2017. V. 63. - P. 119-127.

7. Masahide Inagaki, Ken-ichi Abe An improved anisotropyresolving subgrid-scale model for flows in laminar-turbulent transition region // International Journal of Heat and Fluid Flow. 2017. - V. 64. - P. 137-152.

8. De Marchis M., Milici B., Napoli E. Solid sediment transport in turbulent channel flow over irregular rough boundaries / International Journal of Heat and Fluid Flow. - 2017. -V. 65. P. $114-126$.

9. Sundstrom L.R.J., Cervantes M.J. Laminar similarities between accelerating and decelerating turbulent flows // International journal of heat and fluid flow. -2018 . - V. 71. - P. 13-26. ной поверхности интенсифицируются диффузионные эффекты.

5. Имеется несколько реальных особенностей развития и поддержания напряжений Рейнольдса, корреляций более высокого порядка, характеризующих турбулентную диффузию. В сдвиговом слое при отрыве формируются высокие значения пульсаций нормальных компонент тензора рейнольдсовых напряжений, а затем они снижаются.

Следует отметить, что в настоящем моделировании отсутствуют параметры, позволяющие выполнить надлежащую настройку многопараметрических моделей на расчет сложных течений, за исключением степени турбулентности на входе. Хотя это может составить перспективу использования RANS-метода и его $R S M$-моделей для предсказания статистических динамических параметров, определяющих сложное сдвиговое течение УВ вязких сред в ТП с криволинейной границей, осложненных нестационарностью, неизотермичностью, фазовыми процессами, переменностью теплофизических свойств транспортируемого потока. Более того, дальнейшие исследования в этой области позволят расширить банк данных об особенностях течений в ТП с произвольной геометрией, а также будут способствовать поддержанию эффективных режимов работы, повышению надежности оборудования и систем, применяемых в трубопроводном транспорте.

10. Mi Zhou, Rudi Kulenovic, Eckart Laurien. T-junction experiment with high temperature and high pressure to investigate flow rate influence on mixing characteristics // International Journal of Heat and Fluid Flow. - 2018. - V. 71. - P. 451-459.

11. Глушко Г.С. Дифференциальное уравнение для масштаба турбулентности и расчета турбулентного пограничного слоя на плоской пластине // Турбулентные течения. - М.: Наука, 1970. - C. 37-44.

12. Wilcox D.C. Formulation of the $\mathrm{k} \omega$ turbulence model revisited // AIAA Journal. - 2008. -V. 46. - № 11. - P. 2823-2838.

13. Альгинов Р.А., Харламов С.Н. Ламинаризация газовых потоков в трубопроводах // Горный информационноаналитический бюллетень (научно-технический журнал). 2012. - № OB3. - C. 483-495.

14. Брэдшоу П. Турбулентность. - М.: Машиностроение, 1980. $343 \mathrm{c}$.

15. Фриш У. Турбулентность. Наследие А.Н. Колмогорова. - М.: ФАЗИС, 1998. - $346 \mathrm{c}$.

16. Белоцерковский O.М., Опарин А.М., Чечеткин В.М. Турбулентность: новые подходы. - М.: Наука, 2003. - 286 с.

17. Горбатов А.В., Самуйлов В.Е. О мелкомасштабных квазиупорядоченных структурах в развитой турбулентности // Труды VI Российской национальной конференции по теплообмену. Секция 1. Вынужденная конвекция однофазной жидкости. М.: Изд-во МЭИ, 2006. - Т. 2. - С. 101-104.

18. Eaton J.K., Johnson J.P. Turbulent flow reattachment: an experimental study of the flow and structure behind a backwardfacing step // Report MD-39. Department of Mechanical Engineering. Standard University. -1980 . - P. 1-80.

19. Eaton J.K., Johnston J.P., Jeans A.H. Measurements in a reattaching turbulent shear layer // Proc. of the 2nd Symposium on Turbulent Flows. - London, 1979. - P. 1-13.

20. Narayanan M.A.B., Khadgi Y.N., Viswanath P.R. Similarities in pressure distribution in separated flow behind backward-facing steps // Aeronautical Quarterly. - 1974. - V. 25. - P. 305-312.

21. Kuehn D.M. Some effects of adverse pressure gradient on the incompressible reattaching flow over a rearward-facing step // AIAA Journal. - 1980. - V. 18. - P. 343-344. 
22. De Brederode V., Bradshaw P. Three-dimensional flow in nominally two- dimensional separation of bubbies. I. Behind a rearward-facing step // Aeronautical Report. - 1972. - V. 19. P. $72-91$.

23. Driver D.M., Seegmiller H.L. Features of a reattaching turbulent shear layer in divergent channel flow // AIAA Journal. - 1985. V. 23. - P. 163-171.

24. Summary of the 2004 computational fluid dynamics validation workshop on synthetic jets / C.L. Rumsey, T.B. Gatski, W.L. Sellers, V.N. Vatsa, S.A. Viken // AIAA Journal. - 2006. V. 44. - № 2. - P. 194-207.

25. Balakumar P. DNS/LES simulations of separated flows at high Reynolds number // 49th Fluid Dynamics Conference. AIAA Paper. - 2015. - № 2783. - P. 1-27.

26. Hanjalic K., Launder B.E Contribution towards a Reynolds-stress closure for low-Reynolds-number turbulence // Journal of Fluid Mechanics. - 1976. - V. 74. - Pt. 4. - P. 593-610

27. Spalart P.R., Allmares S.R. A one-equation turbulence model for aerodynamic flows // AIAA Paper. - 1992. - № 0439. - P. 22-36.

28. A new $\mathrm{k}-\varepsilon$ eddy-viscosity model for high Reynolds number turbulent flows - model development and validation / T.-H. Shih, W.W. Liou, A. Shabbir, Z. Yang, J. Zhu // Computers and Fluids. 1995. - V. 24. - № 3. - P. 227-238.

29. Esch T., Menter F.R. Heat transfer predictions based on twoequation turbulence models with advanced wall treatment // Turbultnce, Heat Mass Transfer. - 2003. - № 4. - P. 633-640.
30. Menter F.R. Two-equation eddy-viscosity turbulence models for engineering applications // AIAA Journal. - 1994. - V. 32. - № 8. P. $1598-1605$

31. Walters D.K., Cokljat D. A Three-equation eddy-viscosity model for Reynolds-averaged Navier-Stokes simulations of transitional flow // Journal of Fluids Engineering. - 2008. - V. 130. - № 12. P. 124-131.

32. Correlation based transition model using local variables. P. 1 Model formulation / F.R. Menter, R.B. Langtry, S.R. Likki, Y.B. Suzen, P.G. Huang, S.A. Volker // ASME Journal of Turbomachines. - 2004. - V. 128. - № 3. - P. 413-422.

33. B.E. Launder, Reece G.J., Rodi W. Progress in the development of a Reynolds-stress turbulence closure // Journal of Fluid Mechanics. 1975. - V. 68. - № 3. - P. 537-566.

34. Patankar S.V., Spalding D.B. A Calculation procedure for heat, mass and momentum transfer in three-dimensional parabolic flows // International Journal of the Heat and Mass Transfer. - 1972. V. 15. - P. 1787-1806.

35. Launder B.E., Pridden C.H., Sharma B.I. The calculation of turbulent boundary layers on spinning and curved surfaces // Transactions ASME. Journal of Fluids Engineering. -1977. V. 99. - P. 231-242.

Поступила 16.06.2020 2.

\section{Информация об авторах}

Харламов С.H., доктор физико-математических наук, профессор отделения нефтегазового дела Инженерной школы природных ресурсов Национального исследовательского Томского политехнического университета.

Фатьянов Д.С., аспирант отделения нефтегазового дела Инженерной школы природных ресурсов Национального исследовательского Томского политехнического университета. 
UDC 532.542: 536.242

\title{
INVESTIGATION OF NATURAL RAW MATERIALS TURBULENT FLOW STRUCTURE IN PIPELINES AT CONFUSER-DIFFUSER SECTION
}

\author{
Sergey N. Kharlamov', \\ kharsn@mail.ru \\ Dmitry S. Fatyanov ${ }^{1}$, \\ dsf5@tpu.ru \\ 1 National Research Tomsk Polytechnic University, \\ 30, Lenin avenue, Tomsk, 634050, Russia.
}

The relevance of the research is caused by the need to understand the mechanisms of restructuring hydrodynamic, thermal and diffusion processes accompanying the flow of hydrocarbon viscous media; to explain the nature of their interaction in limited internal areas under the action of complex effects accompanying the flow in complex pipelines; to develop effective solutions for optimal control of natural raw material transport and improvement of reliability of operation of pipelines energy-stressed nodes.

The object of the research is the near-wall flows of drop and gaseous hydrocarbon media in structural elements of internal systems often encountered in engineering applications, in particular, oil and gas, chemical and thermal power industries. Such elements are sections of short or extended pipelines with curved wall surface in the form similar to connecting units or sections of confuser-diffuser type varying in length. Streams are complicated by effects of curvature of flow lines, spatial deformation, changes in dynamic structure and pressure.

The aim of the work is to understand uncertainties, peculiarities and patterns of flows, as well as the possibility of applying modern models of turbulence to forecast the processes in pipelines at confuser-diffuser sections; to study in detail the changes in the «fine» structure of the complex shear flow; to give recommendations in calculating practice of near-wall flows in the specified hydrodynamic configuration and intensive operation modes of the equipment.

Methods: theoretical and practical methods of mathematical modeling and numerical investigation of spatial turbulent flows, peculiarities of changes in their pulsation structure according to schemes (models) with a large number of equations - RANS-models of turbulence, including a dynamic two-parameter base. The choice of the latter depends on the representations: to reproduce statistical correlations or parameters of complex flows, local turbulent properties (integral scale, kinetic energy, dissipation, etc.) of the eddies; to take into account the non-uniform and anisotropic nature of changes in flow structure due to deformation effects excited by variable pressure and Reynolds stresses gradients.

Results. The authors have analyzed the features of turbulent flows of hydrocarbon of viscous media in pipelines including connecting elements of confuser-diffuser type and studied the possibilities of complex shear flows of viscous media, popular in calculations of the pipelines at the curved wall boundary, multi-variable statistical models of second-order turbulence, implemented in high- and low-Reynolds versions. It has been found that a better description of the intensity and size of the recirculation zones of the pipelines is given by the $k-k / \omega / k L-$ and RSM-kL models as being more conservative and resistant to perturbations coming from the walls of the pipeline. Therefore, it is appropriate to include them as the base for the RSM-model. Calculations revealed the existence of a second separated flow zone. It is shown that the greatest changes of turbulent kinetic energy of turbulence, normal components of Reynolds stresses tensor are located in the zone of interaction of shear layer with return and straight-flow separated flow, and the generation is near the separated point. The compliance of calculations of the structure of similar flows with the data of other authors allows stating that understanding of processes, forecast of intensity of dynamic loads in local zones of flow connection to walls according to the presented models will not lead to emergency operation of equipment and will ensure optimal control of transportation of raw materials by pipelines.

\section{Key words:}

Mathematical and numerical modeling, turbulence, hydrodynamics, near-wall flow, pipeline, confuser-diffuser connections.

\section{REFERENCES}

1. Bubenchikov A.M., Kharlamov S.N. Matematicheskie modeli neodnorodnoy anizotropnoy turbulentnosti vo vnutrennikh sistemakh [Mathematical models of inhomogeneous anisotropic turbulence at the internal flows]. Tomsk, Tomsk State University of Publ. House, 2001. 448 p.

2. Kharlamov S.N, Alginov R.A. Specific aspects of turbulent flow separation in nods and junctions of pipelines. IOP Conference Series: Earth and Environmental Science, 2014, vol. 21, pp. 1-5.

3. Kharlamov S.N, Alginov R.A. Validation of second-order statistical models and methods of numerical calculation of the dynamic structure of twisted turbulent flows in pipelines. Izvestiya VUZOV. Physics, 2010, vol. 53, no. 12/2, pp. 267-275. In Rus.

4. Fatyanov D.S. Termodinamicheskie kharakteristiki techeniya gaza $\mathrm{v}$ truboprovode $\mathrm{s}$ konfuzornoy sektsiey [Thermodynamic characteristics of gas flow in a pipeline with a confused section] Trudy XXI Mezhdunarodnogo nauchnogo simpoziuma studentov molodykh uchenykh imeni akademika M.A. Usova. Problemy geologii i osvoeniya nedr [Proc. of the XXI International Scientific Symposium of Students and Young Scientists named after
Academician M.A. Usov. Problems of geology and exploration of mineral resources]. Tomsk, 2017. Vol. 2, pp. 236-239.

5. Terechov V.I., Bogatko T.V., Dyachenko A.Yu., Smulskii Ya.I., Yarigina N.I. Teploobmen $v$ dozvukovykk otryvnykh potokakh [Heat transfer at subsonic separated flows]. Novosobirsk, Novosibirsk State Technical University Publ. House, 2018. 247 p.

6. Howard R.J.A., Serre E. Large eddy simulation in Code_Saturne of thermal mixing in a $\mathrm{T}$ junction with brass walls. International Journal of Heat and Fluid Flow, 2017, vol. 63, pp. 119-127.

7. Masahide Inagaki, Ken-ichi Abe An improved anisotropyresolving subgrid-scale model for flows in laminar-turbulent transition region. International Journal of Heat and Fluid Flow, 2017, vol. 64, pp. 137-152.

8. De Marchis M., Milici B., Napoli E. Solid sediment transport in turbulent channel flow over irregular rough boundaries. International Journal of Heat and Fluid Flow, 2017, vol. 65, pp. 114-126.

9. Sundstrom L.R.J., Cervantes M.J. Laminar similarities between accelerating and decelerating turbulent flows. International journal of heat and fluid flow, 2018, vol. 71, pp. 13-26 . 
10. Mi Zhou, Rudi Kulenovic, Eckart Laurien. T-junction experiment with high temperature and high pressure to investigate flow rate influence on mixing characteristics. International Journal of Heat and Fluid Flow, 2018, vol. 71, pp. 451-459.

11. Glushko G.S. Differentsialnoe uravnenie dlya masshtaba turbulentnosti i raschet turbulentnogo pogranichnogo sloya na ploskoy plastine [Differential equation for turbulence scale and calculation of turbulent boundary layer on flat plate]. Turbulentnye techeniya [Turbulent flows]. Moscow, Nauka Publ., 1970. pp. 34-44.

12. Wilcox D.C. Formulation of the k $\omega$ turbulence model revisited. AIAA Journal, 2008, vol. 46, no. 11, pp. 2823-2838.

13. Alginov R.A., Kharlamov S.N. Laminarization of gas flows in pipelines. Mountain information and analytical bulletin (scientific and technical journal), 2012, no. Spec., Iss. 3, pp. 483-495. In Rus.

14. Bradshaw P. Turbulentnost [Turbulence]. Moscow, Machinostroenie Publ., 1980. 343 p.

15. Frisch U. Turbulentnost. Nasledie A.N. Kolmogorova [Turbulence. A.N. Kolmogorov's heritage]. Moscow, FAZIC Publ., 1998. 346 p.

16. Belotserkovsky O.M., Oparin A.M., Chechen V.M. Turbulentnost: novye pokhody [Turbulence: new approaches]. Moscow, Nauka Publ., 2003. 286 p.

17. Gorbatov A.V., Samuilov V.E. O melkomasshtabnykh kvaziuporyadochenniykh structurakh $\mathrm{v}$ razvitoy turbulentnosti [On small-scale quasi-ordered structures in developed turbulence] Trudy VI Rossiyskoy natsionalnoy konferentsii po teploobmenu. Sektsiya 1. Vynuzhdennaya konvektsiya odnofaznoy zhidkosti [Proc. of the VI Russian National Conference on Heat Exchange. Section 1. Forced convection of single-phase fluid]. Moscow, Moscow Energetic Publ. House, 2006. Vol. 2, pp. 101-104.

18. Eaton J.K., Johnson J.P. Turbulent flow reattachment: an experimental study of the flow and structure behind a backwardfacing step. Report MD-39. Department of Mechanical Engineering. Standard University, 1980. pp. 1-80.

19. Eaton J.K., Johnston J.P., Jeans A.H. Measurements in a reattaching turbulent shear layer. Proc. of the $2^{\text {nd }}$ Symposium on Turbulent Flows. London, 1979, pp. 1-13.

20. Narayanan M.A.B., Khadgi Y.N., Viswanath P.R. Similarities in pressure distribution in separated flow behind backward-facing steps. Aeronautical Quarterly, 1974, vol. 25, pp. 305-312.

21. Kuehn D.M. Some effects of adverse pressure gradient on the incompressible reattaching flow over a rearward-facing step. AIAA Journal, 1980, vol. 18, pp. 343-344.

22. De Brederode V., Bradshaw P. Three-dimensional flow in nominally two- dimensional separation of bubbies. I. Behind a rearward-facing step. Aeronautical Report, 1972, vol. 19, pp. 72-91.
23. Driver D.M., Seegmiller H.L. Features of a reattaching turbulent shear layer in divergent channel flow. AIAA Journal, 1985, vol. 23, pp. $163-171$.

24. Rumsey C.L., Gatski T.B., Sellers W.L., Vatsa V.N., Viken S.A. Summary of the 2004 computational fluid dynamics validation workshop on synthetic jets. AIAA Journal, 2006, vol. 44, no. 2, pp. 194-207.

25. Balakumar P.DNS/LES Simulations of Separated Flows at High Reynolds Number. $49^{\text {th }}$ Fluid Dynamics Conference. AIAA Paper, 2015, no. 2783, pp. 1-27.

26. Hanjalic K., Launder B.E Contribution towards a Reynolds-stress closure for low-Reynolds-number turbulence. Journal of Fluid Mechanics, 1976, vol. 74, Pt. 4, pp. 593-610.

27. Spalart P.R., Allmares S.R. A one-equation turbulence model for aerodynamic flows. AIAA Paper, 1992, no. 0439, pp. 22-36.

28. Shih T.-H., Liou W.W., Shabbir A., Yang Z., Zhu J. A new $k$ - $\varepsilon$ eddy-viscosity model for high Reynolds number turbulent flows model development and validation. Computers and Fluids, 1995, vol. 24, no. 3, pp. 227-238

29. Esch T., Menter F.R. Heat transfer predictions based on twoequation turbulence models with advanced wall treatment. Turbultnce, Heat Mass Transfer, 2003, no. 4, pp. 633-640.

30. Menter F.R. Two-equation eddy-viscosity turbulence models for engineering applications. AIAA Journal, 1994, vol. 32, no. 8, pp. 1598-1605.

31. Walters D.K., Cokljat D. A three-equation eddy-viscosity model for Reynolds-averaged Navier-Stokes simulations of transitional flow. Journal of Fluids Engineering, 2008, vol. 130, no. 12, pp. 124-131.

32. Menter F.R., Langtry R.B., Likki S.R., Suzen Y.B., Huang P.G., Volker S.A. Correlation based transition model using local variables. P. 1 - Model Formulation. ASME Journal of Turbomachines, 2004, vol. 128, no. 3, pp. 413-422.

33. Launder B.E., Reece G.J., Rodi W. Progress in the development of a Reynolds-stress turbulence closure. Journal of Fluid Mechanics, 1975, vol. 68, no. 3, pp. 537-566.

34. Patankar S.V., Spalding D.B. A calculation procedure for heat, mass and momentum transfer in three-dimensional parabolic flows. International Journal of the Heat and Mass Transfer, 1972, vol. 15, pp. 1787-1806.

35. Launder B.E., Pridden C.H., Sharma B.I. The calculation of turbulent boundary layers on spinning and curved surfaces. Transactions ASME. Journal of Fluids Engineering, 1977, vol. 99, pp. 231-242.

Received: 16 June 2020.

\section{Information about the authors}

Sergey N. Kharlamov, Dr. Sc., professor, National Research Tomsk Polytechnic University.

Dmitry S. Fatyanov, postgraduate student, National Research Tomsk Polytechnic University. 